\title{
On Gated Gaussian Impulsive Noise in M-QAM with Optimum Receivers
}

\author{
Évio R. Araújo, Wamberto J. L. Queiroz, Francisco Madeiro, Waslon Terllizzie A. Lopes, and Marcelo S. Alencar
}

\begin{abstract}
This paper presents an analysis of the gated Gaussian impulsive noise and its effects on $M$-ary Quadrature Amplitude Modulation ( $M$-QAM) schemes. In the approach, both amplitude variation and noisy pulse duration can be characterized as a modulation of the impulsive noise component by a discrete (binary or $m$-ary) random process. New exact expressions are presented for the probability density function, autocorrelation function and power spectral density of the noise, as well as for the bit error probability of $M$-QAM, considering the maximum a posteriori probability optimum receiver. An important aspect of the proposed approach is the fact that the discrete random process incorporates the main parameters of the impulsive noise, such as amplitude, duration, instants in which the noise is added and time intervals between instants in which the noise is added.
\end{abstract}

Index Terms-Gated impulsive noise, double gated impulsive noise, digital modulation, bit error probability.

\section{INTRODUCTION}

G ATED Gaussian noise or impulsive interference terminology has been used since 2001, when the Digital Television Group, leaded by BBC R\&D with the participation of Sony, Philips, Rohde \& Schwarz, Zarlink and STMicroelectronics, was created. The purpose of the group was to establish a set of waveforms for tests, and methods to be used to adequately represent the effect of impulsive interference in digital terrestrial television (DTT). A resume of the results of the studies of the group was presented in [1], concerning the contribution of the authors to the group of studies of BBC, from October 2001 to October 2002. Their contribution consisted in the acquisition and statistical analysis of the real impulsive interference for the proposal of a model of noise and conducting measurements in laboratory for validation and simplification of the proposed model.

According to [1], the interference caused by equipment, such as appliances, central heating thermostats, operating switches, rectifiers and ignition systems, occurs in bursts whose duration and intervals between occurrences may be characterized by parameters obtained from measurements. Those bursts, when occur, present amplitude variations that

E. A. Rocha is with Polytechnic School of Pernambuco, University of Pernambuco and Federal Institute of Education, Science and Technology of Pernambuco, Recife, PE, Brazil, e-mail: evio@poli.br.

W. J. L. Queiroz and M. S. Alencar are with Institute for Advanced Studies in Telecommunications, Department of Electrical Engineering, Federal University of Campina Grande, Campina Grande, PB, Brazil, e-mails: \{wamberto,malencar\}@dee.ufcg.edu.br.

F. Madeiro is with Polytechnic School of Pernambuco, University of Pernambuco, Recife, PE, Brazil, e-mail: madeiro@poli.br.

W. T. A. Lopes is with Department of Electrical Engineering, Federal University of Paraíba, João Pessoa, PB, Brazil, e-mail: waslon@ieee.org.

Digital Object Identifier: 10.14209/jcis.2015.2 are statistically characterized by a Gaussian distribution with zero mean and a given variance.

The waveforms presented in [1] can be seen as sample functions of a continuous-time random process, resulting from the modulation by an $m$-ary continuous-time process represented by $C(t)$. The mathematical approach that characterizes the impulsive interference is the main contribution of this research. The advantage that arises from representing the burst behaviour of the impulsive interference as a product of a discrete process $C(t)$ by a white Gaussian process $\eta_{i}(t)$, with zero mean and variance $\sigma_{i}^{2}$, is that some burst aspects, such as duration and time intervals in which they are absent, may be characterized by $C(t)$.

The evaluation of the influence of the impulsive interference on the performance of optimum receivers for M-QAM modulation schemes is also discussed. This evaluation can be performed after the determination of the probability density function of the composed noise $\eta(t)=\eta_{g}(t)+C(t) \eta_{i}(t)$, in which $\eta_{g}(t)$ represents a white Gaussian process, with zero mean and variance $\sigma_{g}^{2}$. This is obtained by expressing the probability density function of the discrete process $C(t)$ in terms of an impulse. Therefore, such model is more general than the one presented in [2], which considers $C(t)$ a Bernoulli process.

In addition to the characterization of the impulsive noise, the paper also addresses its influence on the performance of the maximum a posteriori probability (MAP) receiver for the digital rectangular $M$-QAM ( $M$-ary Quadrature Amplitude Modulation).

Impulsive noise is present in many digital communication systems [3]-[6], and also in Power Line Communication (PLC) networks [7], [8], considering equipment subject to partial discharges [9], [10]. The mitigation of the effects of impulsive noise is important in digital television [11] and OFDM modulation systems [12], [13], wireless communications [14], [15] and development of blind techniques for signal processing in impulsive channels [16].

Concerning digital television channels, GAWGN (Gated Additive White Gaussian Noise) and $\mathrm{G}^{2}$ AWGN (Double Gated Additive White Gaussian Noise) are widely used models, because they are easy to implement and analyze, and they strongly agree with the corresponding experimental measurements [19], [20].

The model was used by the digital television research group at BBC (British Broadcasting Corporation), and allows industry and telecommunications companies to perform impulsive noise simulation. Using those techniques, the development of techniques for mitigating the adverse effects of impulsive noise 
can be accelerated [21].

Proposals for improving DVB-T (Digital Video Broadcasting - Terrestrial) were presented in [22] by using the GAWGN model, with intervals between the pulses fixed in $10 \mathrm{~ms}$, and pulse duration ranging from $10 \mu$ s to $500 \mu \mathrm{s}$. New techniques were presented in [23] to mitigate the impulsive noise in digital television systems using GAWGN.

Recently the GAWGN model was used in the first tests to assess the robustness of DVB-T receivers to impulsive noise [24]. An interesting aspect of the model GAWGN is that it facilitates the simulation of impulsive noise, when compared to other models found in the literature [21].

The GAWGN model represents the occurrence of independent or simple noise pulses, and the $\mathrm{G}^{2} \mathrm{AWGN}$ model is used to represent the noise bursts. Those models can be used to represent impulsive noise present in terrestrial digital television systems. In addition, GAWGN and $\mathrm{G}^{2} \mathrm{AWGN}$ models contribute to the development of noise mitigation techniques and to assess the effects in digital television systems [25].

The International Telecommunication Union (ITU), in the 2008 annual report BT.2035-1, recommends tests to be carried out to assess the performance of digital television systems corrupted by impulsive noise. In order to simulate practical conditions, pulses are generated using the model AWGN for a variety of amplitudes, repetition rates and pulse widths. For each pulse width, the intensity of the noise is increased until the ToV (Threshold of Visibility) of the image is reached [26].

Parameters of the GAWGN and $\mathrm{G}^{2} \mathrm{AWGN}$ models are presented in [19] to simulate impulsive noise due to external reception, internal reception and long duration bursts. Hence, a procedure similar to the one provided by the BBC Digital Television Group (DTG) was used to assess the performance of the Innovative Modulation System Project (MI-SBTVD), developed for the Brazilian Digital Television System, in the presence of impulsive noise.

These categories of impulsive noise are mathematically different from those generated by impulsive electromagnetic discharges from industrial high voltage equipment, as analyzed in [10] and [27], in which the influence is verified on wireless sensor networks communication. Other types of impulsive noise are presented in [28] and modeled as $\alpha$-stable and generalized- $t$, which characterize classes of random processes that arise in practice as a superposition of many independent impulsive effects. In [5] and [6], the impulsive nature of interchannel interference is analyzed in wireless communication systems and the use of non-linear filters for its mitigation is considered.

The remaining of this paper is organized as follows. Section II presents the mathematical characterization of the proposed model for the impulsive noise. The probability density function, the autocorrelation and the power spectrum density for the gated binary Gaussian impulse noise, double gated binary Gaussian impulsive noise, gated multilevel Gaussian impulsive noise and double gated multilevel Gaussian impulsive noise are derived in Sections III, IV, V and VI, respectively. Section VII is devoted to the performance evaluation of MAP receiver with $M$-QAM modulation subject to impulsive noise. Finally, concluding remarks are presented in Section VIII.

\section{Mathematical Characterization of the PROPOSED MODEL}

An approach for the mathematical time domain characterization of impulsive noise models GAWGN and $\mathrm{G}^{2}$ AWGN is presented in this section. The proposed approach uses a digital random modulating signal $C(t)=C_{1}(t) C_{2}(t)$ for amplitude modulation of a white Gaussian noise $\eta_{i}(t)$, with zero mean and variance $\sigma_{i}^{2}$, for characterizing parameters such as amplitude, duration, instants in which the noise is added and time intervals between instants in which the impulsive noise $C(t) \eta_{i}(t)$ is added. The random process $C_{1}(t)$ represents the occurrence of bursts and $C_{2}(t)$ represents the occurrence of pulses. The pulses occur in the interval of duration of the bursts.

The modulating signal $C(t)$ is a binary digital random process, which assumes values in the set $\{0,1\}$, or $m$-ary digital random process, which assumes values in the set $\left\{c_{0}, c_{2}, \cdots, c_{m-1}\right\}$, according to a mass probability function. The total noise in the system is the sum of the impulsive component $C(t) \eta_{i}(t)$ and the permanent noise component represented by a white Gaussian process $\eta_{g}(t)$ of zero mean and variance $\sigma_{g}^{2}$. An important aspect of using a modulating signal $C(t)$ is the fact that parameters of the impulsive noise, such as amplitude, duration, instants in which the noise is added and time intervals between occurrences of pulses and bursts can be modeled by that random process.

In the present work, an additive noise channel is considered in which the received signal $r(t)$ can be written as

$$
r(t)=s(t)+\eta(t),
$$

in which $s(t)$ represents the transmitted signal and

$$
\eta(t)=C_{1}(t) C_{2}(t) \eta_{i}(t)+\eta_{g}(t)
$$

represents the noise composed by an impulsive noisy component $C_{1}(t) C_{2}(t) \eta_{i}(t)$ and by the permanent noisy component $\eta_{g}(t)$. According to the values assumed by $C_{1}(t)$ and $C_{2}(t)$, four different models can be proposed for the noise. In the first model, $C_{1}(t)=1$ and $C_{2}(t)$ randomly assumes values in the set $\{0,1\}$. This model is referred to as Gated Binary Gaussian Impulsive Noise. In the second model, $C_{1}(t)$ and $C_{2}(t)$ randomly assume values in the set $\{0,1\}$. This model is referred to as Double Gated Binary Gaussian Impulsive Noise. In the third model, $C_{1}(t)=1$ and $C_{2}(t)$ randomly assumes values in the discrete set $\left\{c_{20}, c_{21}, \cdots, c_{2\left(m_{2}-1\right)}\right\}$. This model is referred to as Gated Multilevel Gaussian Impulsive Noise. In the last model, $C_{1}(t)$ and $C_{2}(t)$ randomly assume values in the discrete sets $\left\{c_{10}, c_{11}, \cdots, c_{1\left(m_{1}-1\right)}\right\}$ and $\left\{c_{20}, c_{21}, \cdots, c_{2\left(m_{2}-1\right)}\right\}$. This model is referred to as Double Gated Multilevel Gaussian Impulsive Noise.

\section{Gated Binary Gaussian IMPUlsive Noise}

In this model, the impulsive component is obtained multiplying the process $\eta_{i}(t)$ by a square wave $C_{2}(t)$. The time interval in which this square wave assumes unitary value with probability $p_{2}$ is $\alpha T_{2}$, with $0 \leq \alpha \leq 1$. The obtained modulated signal is a sequence of samples of an AWGN process $\eta_{i}(t)$ added to the process which models the permanent noise, 
$\eta_{g}(t)$. The model is referred to as gated because there is no occurrence of noisy bursts. It is referred to as binary because the modulating signal is a binary random sequence of pulses.

The model which describes the behavior of those noise is mathematically represented by

$$
\eta(t)=\eta_{g}(t)+C_{2}(t) \eta_{i}(t)
$$

in which the modulating signal $C_{2}(t)$ randomly assumes amplitudes $m_{l}$ in the set $\{0,1\}$ and can be represented by a sequence of pulses

$$
C_{2}(t)=\sum_{l=-\infty}^{\infty} m_{l} P_{R_{2}}\left(t-l T_{2}\right),
$$

in which $m_{l}$ is a sequence of i.i.d. r.v. with probability distribution $P\left\{m_{l}=1\right\}=p_{2}$ and $P\left\{m_{l}=0\right\}=1-p_{2}$. Additionally, the square pulse $P_{R_{2}}(t)$ of duration $T_{2}$ is defined as

$$
P_{R_{2}}(t)= \begin{cases}1, & 0 \leq t \leq \alpha T_{2} \\ 0, & \text { otherwise }\end{cases}
$$

Thus, the total impulsive noise GAWGN can be expressed as

$$
\eta(t)=\eta_{g}(t)+\left[\sum_{l=-\infty}^{\infty} m_{l} P_{R_{2}}\left(t-l T_{2}\right)\right] \eta_{i}(t) .
$$

Figure 1 presents a realization of that random process obtained from Equation 3.
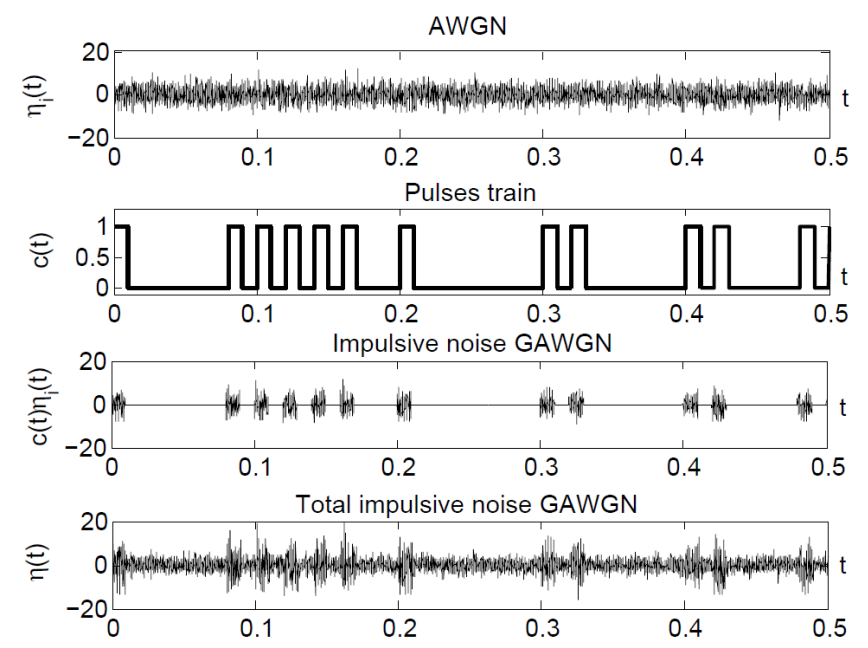

Fig. 1. Realization of the gated binary Gaussian impulsive noise.

\section{A. Probability Density Function}

For obtaining the probability density function (pdf) of the noise in Equation 3, one uses the pdf of the random variable (r.v.) $Y(t)=C_{2}(t) \eta_{i}(t)$. Based on the fact that the pdf of a sum of two independent r.v. is given by the convolution of the individual pdf's [29], the pdf of $\eta(t)$ is obtained by the convolution of the pdf of $\eta_{g}(t)$ with the pdf of $Y(t)$. Thus,

$$
f_{\eta(t)}(\eta)=f_{\eta_{g}(t)}(\eta) * f_{Y}(\eta)
$$

and

$$
\begin{aligned}
f_{\eta(t)}(\eta) & =f_{\eta_{g}(t)}(\eta) *\left[\left(1-p_{2}\right) \delta(\eta)+p_{2} f_{\eta_{i}(t)}(\eta)\right] \\
& =f_{\eta_{g}(t)}(\eta) *\left(1-p_{2}\right) \delta(\eta)+f_{\eta_{g}(t)}(\eta) * p_{2} f_{\eta_{i}(t)}(\eta) \\
& =\left(1-p_{2}\right) f_{\eta_{g}(t)}(\eta)+p_{2} f_{\eta_{g}(t)}(\eta) * f_{\eta_{i}(t)}(\eta) .
\end{aligned}
$$

Substituting the pdf

$$
f_{\eta_{i}(t)}(\eta)=\frac{1}{\sqrt{2 \pi \sigma_{i}^{2}}} \exp \left(-\frac{\eta^{2}}{2 \sigma_{i}^{2}}\right)
$$

and

$$
f_{\eta_{g}(t)}(\eta)=\frac{1}{\sqrt{2 \pi \sigma_{g}^{2}}} \exp \left(-\frac{\eta^{2}}{2 \sigma_{g}^{2}}\right)
$$

in Equation 8 and after some algebraic manipulations, one can write $f_{\eta(t)}(\eta)$ in the form

$$
\begin{aligned}
f_{\eta(t)}(\eta)= & p_{2}\left[\frac{1}{\sqrt{2 \pi\left(\sigma_{g}^{2}+\sigma_{i}^{2}\right)}} \exp \left(-\frac{\eta^{2}}{2\left(\sigma_{g}^{2}+\sigma_{i}^{2}\right)}\right)\right] \\
& +\left(1-p_{2}\right)\left[\frac{1}{\sqrt{2 \pi \sigma_{g}^{2}}} \exp \left(-\frac{\eta^{2}}{2 \sigma_{g}^{2}}\right)\right] .
\end{aligned}
$$

Assuming that $C_{2}(t)=1$ with probability $p_{2}$ in intervals of duration $\alpha T_{2}$ and $C_{2}(t)=0$ with probability $\left(1-p_{2}\right)$ in intervals of duration $(1-\alpha) T_{2}$, the pdf of $\eta(t)$ can be written as

$$
\begin{aligned}
f_{\eta(t)}(\eta)= & \alpha p_{2}\left[\frac{1}{\sqrt{2 \pi\left(\sigma_{g}^{2}+\sigma_{i}^{2}\right)}} \exp \left(-\frac{\eta^{2}}{2\left(\sigma_{g}^{2}+\sigma_{i}^{2}\right)}\right)\right] \\
& +\left(1-\alpha p_{2}\right)\left[\frac{1}{\sqrt{2 \pi \sigma_{g}^{2}}} \exp \left(-\frac{\eta^{2}}{2 \sigma_{g}^{2}}\right)\right] .
\end{aligned}
$$

\section{B. Autocorrelation Function and Power Spectral Density}

The autocorrelation function of the proposed model $\eta(t)$ can be obtained from the definition [30]

$$
\begin{aligned}
R_{\eta}(t, \tau)= & E[\eta(t) \eta(t+\tau)] \\
= & E\left[( \eta _ { g } ( t ) + C _ { 2 } ( t ) \eta _ { i } ( t ) ) \left(\eta_{g}(t+\tau)\right.\right. \\
& \left.\left.+C_{2}(t+\tau) \eta_{i}(t+\tau)\right)\right] .
\end{aligned}
$$

Considering that the processes $\eta_{g}(t)$ and $\eta_{i}(t)$ are zero-mean and independent, one can write $R_{\eta}(t, \tau)$ as

$$
R_{\eta}(t, \tau)=R_{\eta_{g}}(\tau)+E\left[C_{2}(t) C_{2}(t+\tau)\right] R_{\eta_{i}}(\tau),
$$

in which

$$
\begin{aligned}
E\left[C_{2}(t) C_{2}(t+\tau)\right]= & \sum_{l=-\infty}^{\infty} \sum_{k=-\infty}^{\infty}\left[E\left[m_{l} m_{k}\right]\right. \\
& \left.\times P_{R_{2}}\left(t-l T_{2}\right) P_{R_{2}}\left(t+\tau-k T_{2}\right)\right]
\end{aligned}
$$


and $m_{i}, i \in \mathbb{Z}$, are independent and identically Bernoulli random variables with probability distribution $P\left\{m_{i}=1\right\}=p_{2}$ and $P\left\{m_{i}=0\right\}=1-p_{2}$. Therefore 15 can be written as

$$
\begin{aligned}
& E\left[C_{2}(t) C_{2}(t+\tau)\right]= \\
& \underbrace{\sum_{l=-\infty}^{\infty} \sum_{k=-\infty}^{\infty} E\left[m_{l} m_{k}\right] P_{R_{2}}\left(t-l T_{2}\right) P_{R_{2}}\left(t+\tau-k T_{2}\right)}_{k \neq l} \\
& \quad+\sum_{l=-\infty}^{\infty} E\left[m_{l}^{2}\right] P_{R_{2}}\left(t-l T_{2}\right) P_{R_{2}}\left(t+\tau-l T_{2}\right) \\
& =p_{2}^{2} s_{2}(t) s_{2}(t+\tau)+p_{2} \sum_{l=-\infty}^{\infty} P_{R_{2}}\left(t-l T_{2}\right) P_{R_{2}}\left(t+\tau-l T_{2}\right),
\end{aligned}
$$

in which

$$
s_{2}(t)=\sum_{k=-\infty}^{\infty} P_{R_{2}}\left(t-k T_{2}\right)
$$

Applying the result presented in Equation 16 into Equation 14 , one can write

$$
\begin{aligned}
& R_{\eta}(t, \tau)=R_{\eta_{g}}(\tau)+ \\
& \quad+p_{2}^{2} \sum_{l=-\infty}^{\infty} \sum_{k=-\infty}^{\infty} P_{R_{2}}\left(t-l T_{2}\right) P_{R_{2}}\left(t+\tau-k T_{2}\right) \frac{N_{i}}{2} \delta(\tau) \\
& \quad+p_{2} \sum_{l=-\infty}^{\infty} P_{R_{2}}\left(t-l T_{2}\right) P_{R_{2}}\left(t+\tau-l T_{2}\right) \frac{N_{i}}{2} \delta(\tau) .
\end{aligned}
$$

Applying the Fourier transform to $R_{\eta}(t, \tau)$, regarding $\tau$, one can write the power spectral density (PSD) as

$$
\begin{aligned}
S_{\eta}(t, \omega) & =S_{\eta_{g}}(\omega)+\frac{N_{i} p_{2}^{2}}{2} \sum_{l=-\infty}^{\infty} P_{R_{2}}\left(t-l T_{2}\right) \\
& \times \sum_{k=-\infty}^{\infty} \int_{-\infty}^{\infty} P_{R_{2}}\left(t+\tau-k T_{2}\right) \delta(\tau) e^{-j \omega \tau} d \tau \\
& +\frac{p_{2} N_{i}}{2} \sum_{l=-\infty}^{\infty} P_{R_{2}}\left(t-l T_{2}\right) \\
& \times \int_{-\infty}^{\infty} P_{R_{2}}\left(t+\tau-l T_{2}\right) \delta(\tau) e^{-j \omega \tau} d \tau .
\end{aligned}
$$

Applying the sampling property of the Dirac's impulse, one can write the PSD as

$$
\begin{aligned}
S_{\eta}(t, \omega)= & S_{\eta_{g}}(\omega) \\
& +\frac{N_{i} p_{2}^{2}}{2} \underbrace{\sum_{l=-\infty}^{\infty} \sum_{k=-\infty}^{\infty} P_{R_{2}}\left(t-l T_{2}\right) P_{R_{2}}\left(t-k T_{2}\right)}_{k \neq l} \\
& +\frac{p_{2} N_{i}}{2} \sum_{l=-\infty}^{\infty} P_{R_{2}}^{2}\left(t-l T_{2}\right) .
\end{aligned}
$$

The double sum on Equation 20 is null because the train of rectangular pulses are orthogonal when $k \neq l$. For eliminating the dependence of $S_{\eta}(t, \omega)$ with respect to the time $t$, one can take the temporal mean, so

$$
S_{\eta}(\omega)=\lim _{T \rightarrow \infty} \frac{1}{T} \int_{-T / 2}^{T / 2} S_{\eta}(t, \omega) d t=\frac{N_{0}}{2}+p_{2} \alpha \frac{N_{i}}{2} .
$$

\section{Double Gated Binary Gaussian Impulsive Noise}

In this model, the process AWGN $\eta_{g}(t)$, of zero mean and variance $\sigma_{g}^{2}$, is added to the process AWGN $\eta_{i}(t)$, of zero mean and variance $\sigma_{i}^{2}$, and multiplied by two modulating binary signals, $C_{1}(t)$ and $C_{2}(t)$, which are formed by rectangular pulses of duration $T_{1}$ and $T_{2}$, respectively. The time interval in which $C_{1}(t)$ assumes unitary value is $\beta T_{1}$ and the time interval in which $C_{2}(t)$ assumes unitary value is $\alpha T_{2}$.

Considering $b$ pulses in each burst, $T_{1}=b T_{2}$. The modulated noise obtained, $\eta_{i}(t) C_{1}(t) C_{2}(t)$, forms a sequence of noisy bursts with duration $\beta T_{1}$, in which each pulse has duration $\alpha T_{2}$. The modulating signals $C_{1}(t)$ and $C_{2}(t)$ randomly assume the values $m_{k}$ and $m_{l}$, respectively, in the set $\{0,1\}$.

Thus, the model which describes the behavior of that noise is given by

$$
\eta(t)=\eta_{g}(t)+C_{1}(t) C_{2}(t) \eta_{i}(t)
$$

in which the modulating signal $C_{1}(t)$, which is associated with the presence or absence of bursts, is represented by the sequence of rectangular pulses

$$
C_{1}(t)=\sum_{k=-\infty}^{\infty} m_{k} P_{R_{1}}\left(t-k T_{1}\right),
$$

in which the probability distribution of $m_{k}$ is given by $P\left\{m_{k}=1\right\}=p_{1}$ and $P\left\{m_{k}=0\right\}=1-p_{1}$.

The modulating signal $C_{2}(t)$, which is associated to the presence or absence of pulses, is represented by the sequence of rectangular pulses

$$
C_{2}(t)=\sum_{l=-\infty}^{\infty} m_{l} P_{R_{2}}\left(t-l T_{2}\right),
$$

in which the probability distribution of $m_{l}$ is given by $P\left\{m_{l}=\right.$ $1\}=p_{2}$ and $P\left\{m_{l}=0\right\}=1-p_{2}$.

In both modulating signals, the rectangular pulses $P_{R_{1}}(t)$ and $P_{R_{2}}(t)$, of duration $T_{1}$ and $T_{2}$, are given by

$$
P_{R_{1}}(t)= \begin{cases}1, & 0 \leq t \leq \beta T_{1} \\ 0, & \text { otherwise }\end{cases}
$$

in which $0 \leq \beta \leq 1$, and by

$$
P_{R_{2}}(t)= \begin{cases}1, & 0 \leq t \leq \alpha T_{2} \\ 0, & \text { otherwise }\end{cases}
$$

in which $0 \leq \alpha \leq 1$.

Thus, the total noise can be given by

$$
\begin{aligned}
& \eta(t)=\eta_{g}(t)+C_{1}(t) C_{2}(t) \eta_{i}(t) \\
& =\eta_{g}(t)+\sum_{k=-\infty}^{\infty} \sum_{l=-\infty}^{\infty} m_{k} m_{l} P_{R_{1}}\left(t-k T_{1}\right) P_{R_{2}}\left(t-l T_{2}\right) \eta_{i}(t) .
\end{aligned}
$$

Figure 2 presents a realization of that random process from Equation 22. 


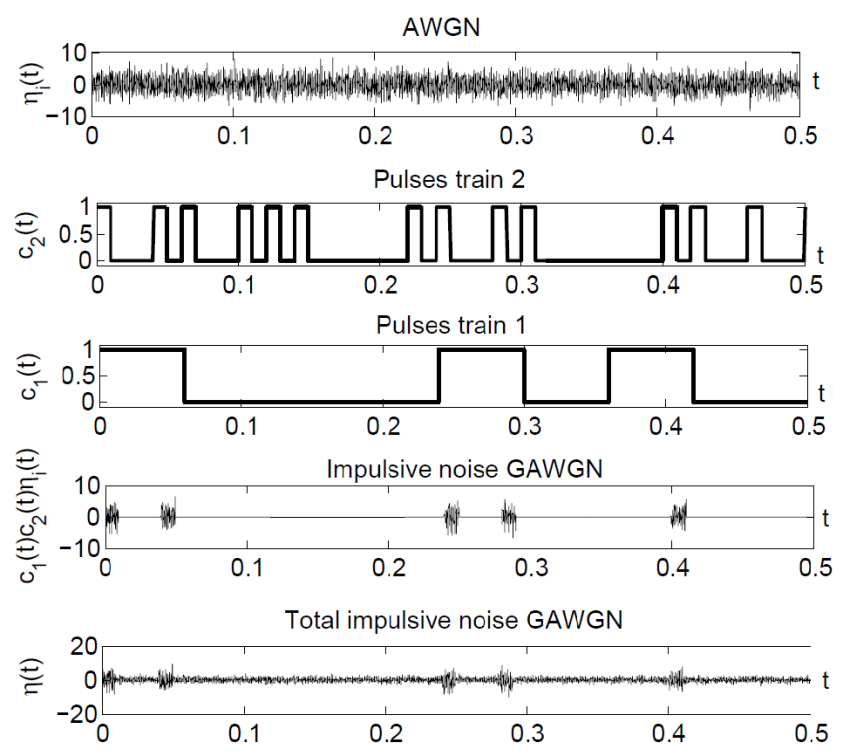

Fig. 2. Realization of the double gated binary Gaussian impulsive noise.

\section{A. Probability Density Function}

The procedure for determining the pdf of the process $\eta(t)$ which represents the double gated binary Gaussian impulsive noise is similar to the one used in Section III-A. In this second model, both $C_{1}(t)$ and $C_{2}(t)$ switch and assume random values in the set $\{0,1\}$. Hence, the joint probability density function of $C_{1}(t)$ and $C_{2}(t)$ can be written as

$$
f_{C_{1}(t), C_{2}(t)}\left(c_{1}, c_{2}\right)=\sum_{k=0}^{1} \sum_{l=0}^{1} p_{1 k} p_{2 l} \delta\left(c-c_{1 k} c_{2 l}\right)
$$

in which the mass probability functions (mpf) of the values assumed by $C_{1}(t)$ and $C_{2}(t)$ in the sets $\left\{c_{10}, c_{11}, \cdots, c_{1\left(m_{1}-1\right)}\right\}$ and $\left\{c_{20}, c_{11}, \cdots, c_{1\left(m_{2}-1\right)}\right\}$ are given by $P\left\{C_{1}(t)=c_{1 k}\right\}=$ $p_{1 k}$ and $P\left\{C_{2}(t)=c_{2 l}\right\}=p_{2 l}$.

Thus, the expression of the total noise $\eta(t)$ can be written as

$$
\eta(t)=\eta_{g}(t)+C_{1}(t) C_{2}(t) \eta_{i}(t)
$$

Then, the pdf of $\eta(t)$ can be written as

$$
\begin{aligned}
f_{\eta(t)}(\eta)=\sum_{k=0}^{1} \sum_{l=0}^{1} & \frac{p_{1 k} p_{2 l}}{\sqrt{2 \pi\left(\sigma_{g}^{2}+\sigma_{i}^{2} c_{1 k}^{2} c_{2 l}^{2}\right)}} \\
& \times \exp \left(-\frac{\eta^{2}}{2\left(\sigma_{g}^{2}+\sigma_{i}^{2} c_{1 k}^{2} c_{2 l}^{2}\right)}\right) .
\end{aligned}
$$

Considering that the pulses $P_{R_{1}}(t)$ and $P_{R_{2}}(t)$, present in the modulating signals $C_{1}(t)$ e $C_{2}(t)$, assume unitary values in the intervals of $\beta T_{1}$ and $\alpha T_{2}$, with probabilities $p_{1} \mathrm{e}$ $p_{2}$, respectively, one can write the pdf of $\eta(t)$, given in
Equation 30, as

$$
\begin{aligned}
f_{\eta(t)}(\eta)= & \frac{\alpha \beta p_{1} p_{2}}{\sqrt{2 \pi\left(\sigma_{g}^{2}+\sigma_{i}^{2}\right)}} \exp \left[-\frac{\eta^{2}}{2\left(\sigma_{g}^{2}+\sigma_{i}^{2}\right)}\right] \\
& +\frac{\left(1-\alpha \beta p_{1} p_{2}\right)}{\sqrt{2 \pi \sigma_{g}^{2}}} \exp \left[-\frac{\eta^{2}}{2 \sigma_{g}^{2}}\right] .
\end{aligned}
$$

\section{B. Autocorrelation Function and Power Spectral Density}

The autocorrelation function is obtained from $\eta(t)$ given in Equation 22 and can be expressed as

$$
R_{\eta}(t, \tau)=E[\eta(t) \eta(t+\tau)] .
$$

Considering that the processes $\eta_{g}(t)$ and $\eta_{i}(t)$ are independent and the probability distributions for $m_{k}$ and $m_{l}$, of the signals $C_{1}(t)$ and $C_{2}(t)$ given in Equations 23 and 24, one can write $R_{\eta}(t, \tau)$ as

$$
R_{\eta}(t, \tau)=\frac{N_{0}}{2} \delta(\tau)+s_{12}(t) p_{1} p_{2} \frac{N_{i}}{2} \delta(\tau)
$$

in which

$$
s_{12}(t)=\sum_{k=-\infty}^{\infty} \sum_{l=-\infty}^{\infty} P_{R_{1}}\left(t-k T_{1}\right) P_{R_{2}}\left(t-l T_{2}\right) .
$$

For eliminating the dependence of the autocorrelation with respect to time $t$, one can obtain the time mean of $R_{\eta}(t, \tau)$, as

$$
\begin{aligned}
R_{\eta}(\tau) & =\lim _{T_{1} \rightarrow \infty} \frac{1}{T_{1}} \int_{-T_{1} / 2}^{T_{1} / 2} R_{\eta}(t, \tau) d t \\
& =\frac{N_{0}}{2} \delta(\tau)+p_{1} p_{2} \alpha \beta \frac{N_{i}}{2} \delta(\tau) .
\end{aligned}
$$

The PSD of $\eta(t)$ is obtained by the Fourier transform of the autocorrelation function $R_{\eta}(\tau)$, that is

$$
\begin{aligned}
S_{\eta}(\omega) & =\int_{-\infty}^{\infty} R_{\eta}(\tau) \exp (-j \omega \tau) d \tau \\
& =\frac{N_{0}}{2}+\alpha \beta p_{1} p_{2} \frac{N_{i}}{2} .
\end{aligned}
$$

\section{Gated Multilevel Gaussian Impulsive Noise}

In this model, $C_{2}(t)$ can assume values in the discrete set $\left\{c_{20}, c_{21}, \cdots, c_{2\left(m_{2}-1\right)}\right\}$. The modulating signal $C_{2}(t)$ characterizes both the intensity variation of $\eta_{i}(t)$ and the instants in which $\eta_{i}(t)$ is added to the permanent noise represented by the random process $\eta_{g}(t)$. The signal $C_{2}(t)$ is a discrete random process characterized by a probability distribution that can be defined in both discrete time and continuous time.

The model that describes the behavior of such noise is given by

$$
\eta(t)=\eta_{g}(t)+C_{2}(t) \eta_{i}(t)
$$

in which $C_{2}(t)$ is characterized by the pdf

$$
f_{C_{2}(t)}(c)=\sum_{l} p_{2 l} \delta\left(c-c_{2 l}\right),
$$

in which $p_{2 l}=P\left\{C_{2}(t)=c_{2 l}\right\}$.

Figure 3 represents a realization of the random process $\eta(t)$ as a function of time, from Equation 37. 

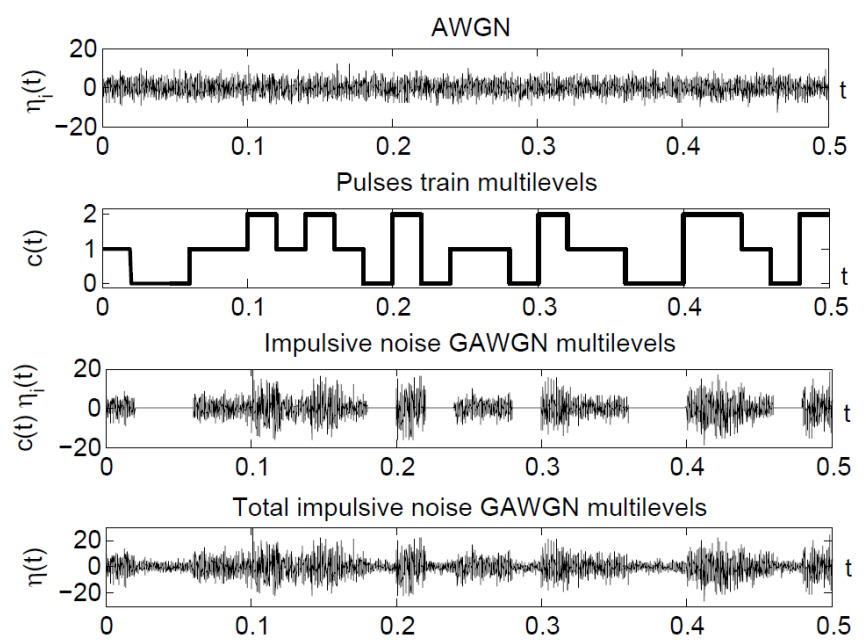

Fig. 3. Realization of the gated multilevel Gaussian impulsive noise.

\section{A. Probability Density Function}

The procedure for obtaining the pdf of $\eta(t)$ is similar to the one used to obtain the pdf of the first two models. In this model, since $\eta_{i}(t) C_{2}(t)$ and $\eta_{g}(t)$ are independent, the pdf of $\eta(t)$ can be written as

$$
f_{\eta(t)}(\eta)=\sum_{l=0}^{m_{2}-1} \frac{p_{2 l}}{\sqrt{2 \pi\left(\sigma_{g}^{2}+\sigma_{i}^{2} c_{2 l}^{2}\right)}} \exp \left(-\frac{\eta^{2}}{2\left(\sigma_{g}^{2}+\sigma_{i}^{2} c_{2 l}^{2}\right)}\right) .
$$

One can observe, from Equation 39, that the pdf of $\eta(t)$ depends on values assumed by the modulating signal at instant $t$. If the process $C_{2}(t)$ assumes only two values, like random binary signals, then that sum only has two terms. Other important aspect of $\eta(t)$ is that its pdf remains with the format of a symmetrical Gaussian pdf, since both $\eta_{g}(t)$ and $\eta_{i}(t)$ are zero mean.

The pdf of $\eta(t)$ can also be written as

$$
\begin{aligned}
f_{\eta(t)}(\eta) & =p_{20} f_{c_{20}}(\eta)+p_{21} f_{c_{21}}(\eta) \\
& +p_{23} f_{c_{33}}(\eta)+\ldots+p_{2\left(m_{2}-1\right)} f_{c_{2\left(m_{2}-1\right)}}(\eta),
\end{aligned}
$$

in which

$$
f_{c_{2 l}}(\eta)=\frac{1}{\sqrt{2 \pi\left(\sigma_{g}^{2}+\sigma_{i}^{2} c_{2 l}^{2}\right)}} \exp \left(-\frac{\eta^{2}}{2\left(\sigma_{g}^{2}+\sigma_{i}^{2} c_{2 l}^{2}\right)}\right) .
$$

Equation 40 is a Gaussian mixture formed by probability density functions of zero mean and variance $\sigma_{g}^{2}+\sigma_{i}^{2} c_{2 l}^{2}$. The constants $c_{2 l}$ correspond to the values the signal $C_{2}(t)$ can assume. In Figure 3, for instance, $C_{2}(t)$ assume three equiprobable values, $c_{20}=0, c_{21}=1$ and $c_{22}=2$.

Considering the probability distributions $p_{20}, p_{21}, \cdots$, $p_{2\left(m_{2}-1\right)}$, respectively, of the levels $c_{20}, c_{21}, \cdots, c_{2\left(m_{2}-1\right)}$ of the multilevel modulating signal $C_{2}(t)$ and the duration $\alpha T_{2}$ of the rectangular pulses $P_{R_{2}}(t)$ present in $C_{2}(t)$, one can write the pdf of $\eta(t)$, given in Equation 39, as

$$
f_{\eta(t)}(\eta)=\alpha \sum_{l=0}^{m_{2}-1} p_{2 l} f_{c_{2 l}}(\eta)+(1-\alpha) f_{\eta_{g}(t)}(\eta)
$$

in which $p_{2 l}=P\left\{C_{2}(t)=c_{2 l}\right\}$.

\section{B. Autocorrelation Function and Power Spectral Density}

The autocorrelation function is obtained from $\eta(t)$, in Equation 37 , and can be written as

$$
\begin{aligned}
R_{\eta}(t, \tau) & =R_{\eta_{g}}(\tau)+R_{\eta_{i}}(\tau) s_{2}(t) \sum_{l=0}^{m_{2}-1} p_{2 l} c_{2 l}^{2} \\
& =\frac{N_{0}}{2} \delta(\tau)+\frac{N_{i}}{2} \delta(\tau) s_{2}(t) \sum_{l=0}^{m_{2}-1} p_{2 l} c_{2 l}^{2},
\end{aligned}
$$

in which $s_{2}(t)=\sum_{l=-\infty}^{\infty} P_{R_{2}}\left(t-l T_{2}\right)$. For eliminating the dependence of the autocorrelation function with respect to time $t$, one can obtain the time mean of $R_{\eta}(t, \tau)$, as

$$
R_{\eta}(\tau)=\alpha \frac{N_{0}}{2} \delta(\tau)+\alpha \frac{N_{i}}{2} \delta(\tau) \sum_{l=0}^{m_{2}-1} p_{2 l} c_{2 l}^{2} .
$$

Then, the power spectral density can be written as

$$
S_{\eta}(\omega)=\alpha \frac{N_{0}}{2}+\alpha \frac{N_{i}}{2} \sum_{l=0}^{m_{2}-1} p_{2 l} c_{2 l}^{2}
$$

\section{Vi. Double Gated Multilevel Gaussian Impulsive NOISE}

In this model, the modulating signals $C_{1}(t)$ and $C_{2}(t)$ can assume non-negative discrete values in the sets $\left\{c_{10}, c_{11}, \cdots, c_{1\left(m_{1}-1\right)}\right\}$ and $\left\{c_{20}, c_{21}, \cdots, c_{2\left(m_{2}-1\right)}\right\}$. The random modulating signal $C(t)=C_{1}(t) C_{2}(t)$ is used to randomly change the power of the impulsive noise $\eta_{i}(t)$ added to the permanent Gaussian noise.

The model that describes the behavior of such noise is expressed as

$$
\eta(t)=\eta_{g}(t)+C_{1}(t) C_{2}(t) \eta_{i}(t)
$$

in which the signals $C_{1}(t)$ and $C_{2}(t)$ are given respectively by

$$
C_{1}(t)=\sum_{k=-\infty}^{\infty} m_{1 k} P_{R_{1}}\left(t-k T_{1}\right)
$$

and

$$
C_{2}(t)=\sum_{l=-\infty}^{\infty} m_{2 l} P_{R_{2}}\left(t-l T_{2}\right)
$$

The joint pdf of $C_{1}(t)$ and $C_{2}(t)$ can be written as

$$
f_{C_{1}(t), C_{2}(t)}\left(c_{1}, c_{2}\right)=\sum_{k=0}^{m_{1}-1} \sum_{l=0}^{m_{2}-1} p_{1 k} p_{2 l} \delta\left(c-c_{1 k} c_{2 l}\right) .
$$

Figure 4 presents a realization of the process $\eta(t)$ obtained from Equation 46. One can observe the random behavior of the amplitudes and the duration of the bursts. 

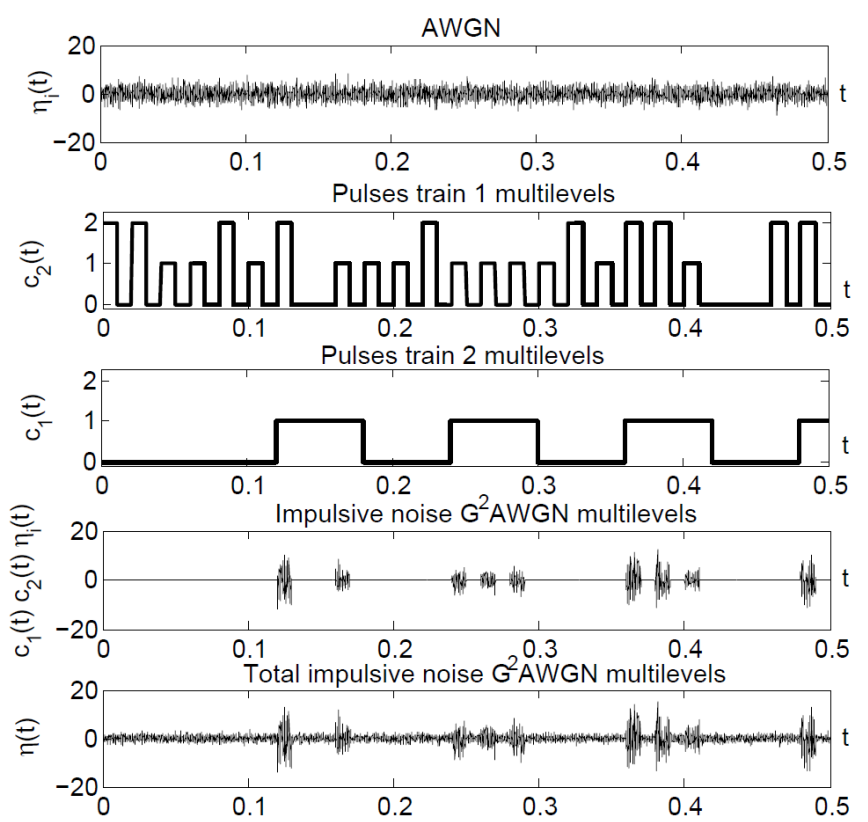

Fig. 4. Realization of the double gated multilevel Gaussian impulsive noise.

\section{A. Probability Density Function}

Following a procedure similar to the one used in previous sections concerning the other models of noise, one can write the pdf of the process that models the double gated multilevel Gaussian impulsive noise as

$$
\begin{aligned}
f_{\eta(t)}(\eta)= & \sum_{k=0}^{m_{1}-1} \sum_{l=0}^{m_{2}-1} \frac{p_{1 k} p_{2 l}}{\sqrt{2 \pi\left(\sigma_{g}^{2}+\sigma_{i}^{2} c_{1 k}^{2} c_{2 l}^{2}\right)}} \\
& \times \exp \left(-\frac{\eta^{2}}{2\left(\sigma_{g}^{2}+\sigma_{i}^{2} c_{1 k}^{2} c_{2 l}^{2}\right)}\right) .
\end{aligned}
$$

Considering the probability distributions $p_{10}, p_{11}, \cdots$, $p_{1\left(m_{1}-1\right)}$ and $p_{20}, p_{21}, \cdots, p_{2\left(m_{2}-1\right)}$ respectively of the levels $c_{10}, c_{11}, \cdots, c_{1\left(m_{1}-1\right)}$ and $c_{20}, c_{21}, \cdots, c_{2\left(m_{2}-1\right)}$, as well as the durations $\beta T_{1}$ and $\alpha T_{2}$ of the rectangular pulses $P_{R_{1}}(t)$ and $P_{R_{2}}(t)$ present in $C_{1}(t)$ and $C_{2}(t)$, the pdf $\eta(t)$ can be written as

$$
f_{\eta(t)}(\eta)=\alpha \beta \sum_{k=0}^{m_{1}-1} \sum_{l=0}^{m_{2}-1} p_{1 k} p_{2 l} f_{k l}(\eta)+(1-\alpha \beta) f_{\eta_{g}}(\eta)
$$

in which

$$
f_{k l}(\eta)=\frac{1}{\sqrt{2 \pi\left(\sigma_{g}^{2}+\sigma_{i}^{2} c_{1 k}^{2} c_{2 l}^{2}\right)}} \exp \left(-\frac{\eta^{2}}{2\left(\sigma_{g}^{2}+\sigma_{i}^{2} c_{1 k}^{2} c_{2 l}^{2}\right)}\right) .
$$

\section{B. Autocorrelation Function and Power Spectral Density}

The autocorrelation function of the process that models the double gated multilevel Gaussian impulsive noise, given in
Equation 46, can be written as

$$
R_{\eta}(t, \tau)=\frac{N_{0}}{2} \delta(\tau)+\frac{N_{i}}{2} \delta(\tau) s(t) \sum_{k=0}^{m_{1}-1} \sum_{l=0}^{m_{2}-1} p_{1 k} p_{2 l} c_{1 k}^{2} c_{2 l}^{2},
$$

in which $s_{12}(t)=\sum_{k=-\infty}^{\infty} \sum_{l=-\infty}^{\infty} P_{R_{1}}\left(t-k T_{1}\right) P_{R_{2}}\left(t-l T_{2}\right)$. For eliminating the dependence of the autocorrelation function with respect to time $t$, one can calculate the time mean of $R_{\eta}(t, \tau)$ as

$$
R_{\eta}(\tau)=\frac{N_{0}}{2} \delta(\tau)+\alpha \beta \frac{N_{i}}{2} \delta(\tau) \sum_{k=0}^{m_{1}-1} \sum_{l=0}^{m_{2}-1} p_{1 k} p_{2 l} c_{1 k}^{2} c_{2 l}^{2} .
$$

The power spectral density can then be written as

$$
S_{\eta}(\omega)=\frac{N_{0}}{2}+\alpha \beta \frac{N_{i}}{2} \sum_{k=0}^{m_{1}-1} \sum_{l=0}^{m_{2}-1} p_{1 k} p_{2 l} c_{1 k}^{2} c_{2 l}^{2} .
$$

\section{ViI. Performance Evaluation of MAP Receiver} With Modulation $M$-QAM Under IMPUlsive NoIsE

In this section, four models of impulsive noise, presented in Sections III, IV, V and VI, are considered. A performance evaluation of MAP receiver [31] under impulsive noise is carried out. For the evaluation, square $M$-QAM modulation is considered and the bit error probability (BEP) is derived from the expressions introduced in [32].

According to Cho and Yoon [32], the BEP of $M$-QAM, given a signal-to-noise ratio $\mathrm{SNR} \gamma=E_{b} / N_{0}$, can be written, in terms of the constellation order, $M$, as

$$
P_{M}(e \mid \gamma)=\frac{1}{\log _{2} \sqrt{M}} \sum_{k=1}^{\log _{2} \sqrt{M}} P_{b}(k)
$$

in which $P_{b}(k)$ can be written as

$$
\begin{aligned}
P_{b}(k)=\frac{1}{\sqrt{M}} & \sum_{i=0}^{\left(1-2^{-k}\right) \sqrt{M}-1}\{w(i, k, M) \\
& \left.\times \operatorname{erfc}\left((2 i+1) \sqrt{\frac{3 \log _{2} M \gamma}{2(M-1)}}\right)\right\},
\end{aligned}
$$

the coefficients $w(i, k, M)$ are given by

$$
w(i, k, M)=(-1)^{\left\lfloor\frac{i 2^{k-1}}{\sqrt{M}}\right\rfloor} \cdot\left(2^{k-1}-\left\lfloor\frac{i \cdot 2^{k-1}}{\sqrt{M}}+\frac{1}{2}\right\rfloor\right)
$$

and the term $\lfloor x\rfloor$ denotes the largest integer smaller than or equal to $x$. The term

$$
\operatorname{erfc}\left((2 i+1) \sqrt{\frac{3 \log _{2} M E_{b}}{2(M-1)}}\right)
$$

in Equation 57, when written in terms of the $Q(\cdot)$ function,

$$
\begin{aligned}
\operatorname{erfc}\left((2 i+1) \sqrt{\frac{3 \log _{2} M E_{b}}{2(M-1)}}\right)= \\
2 \mathrm{Q}\left(\sqrt{2}(2 i+1) \sqrt{\frac{3 \log _{2} M E_{b}}{2(M-1)}}\right),
\end{aligned}
$$


can be seen as twice the probability that the noise exceeds

$$
(2 i+1) \sqrt{\frac{3 \log _{2} M E_{b}}{(M-1)}} .
$$

Thus, for the proposed impulsive noise models, that probability, given by

$$
2 \operatorname{Prob}\left\{\eta \geq(2 i+1) \sqrt{\frac{3 \log _{2} M E_{b}}{(M-1)}}\right\},
$$

can be obtained by the integration of the pdf of the process that represents the noise, $\eta(t)$, in the interval $\left[(2 i+1) \sqrt{\frac{3 \log _{2} M E_{b}}{(M-1)}}, \infty\right)[31],[32]$. Then, one can write the probability $P_{b}(k)$ for each one of the models presented in Sections III, IV, V and VI. Hence, substituting those probabilities in Expression 56, one obtain the expressions for the BEP of each one of the proposed models. It is worth to mention that the authors have successfully used this approach to determine the BEP of modulation schemes in Nakagami- $m$, $\eta-\mu$ and $\kappa-\mu$ fading channels [15], [17], [18]

\section{A. BEP of the Gated Binary Gaussian Impulsive Noise}

For the gated binary Gaussian impulsive noise, presented in Section III, the probability $P_{b}(k)$ can be written as

$$
\begin{aligned}
P_{b}(k)= & \frac{2}{\sqrt{M}} \sum_{i=0}^{\left(1-2^{-k}\right) \sqrt{M}-1} w(i, k, M) \\
& \times\left(\alpha p_{2} \mathrm{Q}\left(\sqrt{a(i, M) \frac{\gamma_{g} \gamma_{i}}{\gamma_{g}+\gamma_{i}}}\right)\right. \\
& \left.+\left(1-\alpha p_{2}\right) \mathrm{Q}\left(\sqrt{a(i, M) \gamma_{g}}\right)\right),
\end{aligned}
$$

in which $a(i, M)=\frac{3(2 i+1)^{2} \log _{2}(M)}{(M-1)}, \gamma_{g}=E_{b} / N_{0}$ represents the signal-to-permanent noise ratio and the term $\gamma_{i}=E_{b} / N_{i}$ will be referred to as signal-to-impulsive noise ratio, even knowing that the impulsive noise, in the general case, is modeled by the product $C_{1}(t) C_{2}(t) \eta_{i}(t)$.

Figure 5 shows BEP curves for 64-QAM under gated binary Gaussian impulsive noise for different values of signal-toimpulsive noise ratio $\gamma_{i}=\frac{E_{b}}{N_{i}}$. The probability distribution of the levels of amplitudes of $C_{2}(t)$ and the value of the parameter $\alpha$ are given by

$$
\left\{\begin{array}{l}
P\left\{C_{2}(t)=1\right\}=p_{2}=0.7 \\
P\left\{C_{2}(t)=0\right\}=1-p_{2}=0.3 \\
\alpha=0.5
\end{array} .\right.
$$

In Expression 64, $\alpha=0.5$ is the percentage of the duration $T_{2}$, of the pulses $P_{R_{2}}(t)$ of $C_{2}(t)$, in which $C_{2}(t)$ assumes unitary value. In this interval, the noise $\eta_{i}(t)$ is added to the permanent noise with probability $p_{2}$ or is absent with probability $1-p_{2}$. The case in which $p_{2}=0.7$ characterizes a model in which the noisy pulses are present more frequently. It is observed in the curves of Figure 5 that when the signalto-permanent noise reaches values greater than the signal-toimpulsive noise ratio $\gamma_{i}$, for fixed values of $\gamma_{i}$, the BEP tends to decrease. This occurs because when $\gamma_{g}>\gamma_{i}$ the energy of

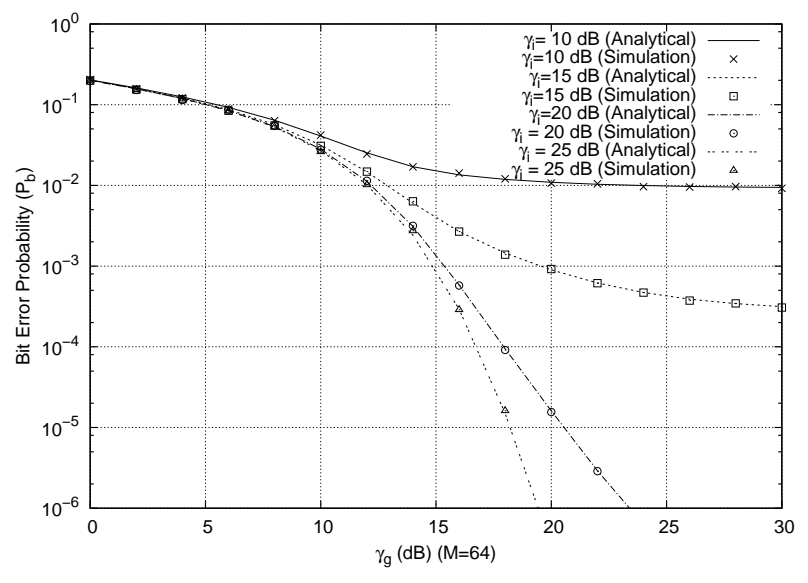

Fig. 5. BEP of 64-QAM under gated binary Gaussian impulsive noise.

the permanent noise $\eta_{g}(t)$ is smaller than the energy of the impulsive noise $C_{2}(t) \eta_{i}(t)$, which acts less frequently.

A behavior observed in the curves, in accordance with the BEP equations presented in this paper, is the tendency that the curves associated to the smaller value of $\gamma_{i}$ remain constant with the increase of $\gamma_{g}$. When $\gamma_{g}$ is much greater than $\gamma_{i}$, one of the functions $Q(x)$ depends more on $\gamma_{i}$ while the other function $Q(x)$, which depends only on $\gamma_{g}$, approaches zero. This explains the reason why the BEP tends to remain constant.

\section{B. BEP of the Double Gated Binary Gaussian Impulsive Noise}

For the double gated binary Gaussian impulsive noise, presented in Section IV, the probability $P_{b}(k)$ can be written as

$$
\begin{aligned}
P_{b}(k)= & \frac{2}{\sqrt{M}} \sum_{i=0}^{\left(1-2^{-k}\right) \sqrt{M}-1} w(i, k, M) \\
& \left\{\alpha \beta p_{1} p_{2} \mathrm{Q}\left(\sqrt{a(i, M) \frac{\gamma_{g} \gamma_{i}}{\gamma_{g}+\gamma_{i}}}\right)\right. \\
& \left.+\left(1-\alpha \beta p_{1} p_{2}\right) \mathrm{Q}\left(\sqrt{a(i, M) \gamma_{g}}\right)\right\} .
\end{aligned}
$$

Figure 6 presents BEP curves for 64-QAM under double gated binary Gaussian impulsive noise, for different values of the signal-to-impulsive noise ratio, $\gamma_{i}=\frac{E_{b}}{N_{i}}$. The probability distributions of the levels of amplitude of the signals $C_{1}(t)$ and $C_{2}(t)$ and the values of the parameters $\alpha$ and $\beta$ are given by

$$
\left\{\begin{array}{l}
P\left\{C_{1}(t)=1\right\}=p_{1}=0.25 \\
P\left\{C_{1}(t)=0\right\}=1-p_{1}=0.75 \\
\alpha=0.5
\end{array}\right.
$$

and

$$
\left\{\begin{array}{l}
P\left\{C_{2}(t)=1\right\}=p_{2}=0.75 \\
P\left\{C_{2}(t)=0\right\}=1-p_{2}=0.25 \\
\beta=0.5 .
\end{array}\right.
$$

In Expressions 67 and 66, $\alpha=0.5$ and $\beta=0.5$ represent, respectively, the percentage of the durations $T_{1}$ and $T_{2}$ of the pulses $P_{R_{1}}(t)$ and $P_{R_{2}}(t)$, of the modulating signals $C_{1}(t)$ and 


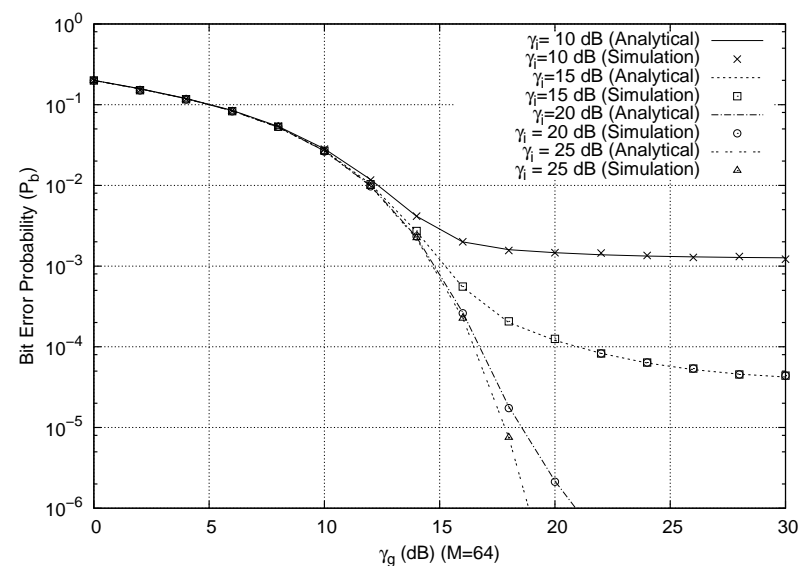

Fig. 6. BEP of 64-QAM under double gated binary Gaussian impulsive noise.

$C_{2}(t)$, in which those signals assume unitary values. In those intervals, the impulsive noise $\eta_{i}(t)$ is added to the permanent noise with probability $p_{1} p_{2}$ or is absent with probability $1-$ $p_{1} p_{2}$. The curves in Figure 6 present a behavior similar to the ones of Figure 5.

An aspect of Figure 6 to be pointed out, with respect for instance to the curve of $\gamma_{i}=10 \mathrm{~dB}$, is that when $\gamma_{i}$ is kept constant and much smaller than $\gamma_{g}$ (case in which the energy of the impulsive noise is higher than the energy of the permanent noise), the BEP does not decrease with the increase of $\gamma_{g}$.

\section{BEP of the Gated Multilevel Gaussian Impulsive Noise}

For the gated multilevel Gaussian impulsive noise, presented in Section $\mathrm{V}$, the probability $P_{b}(k)$ can be written as

$$
\begin{aligned}
P_{b}(k)= & \frac{2}{\sqrt{M}} \sum_{i=0}^{\left(1-2^{-k}\right) \sqrt{M}-1} w(i, k, M) \times \\
& \left\{\alpha \sum_{l=0}^{m_{2}-1} p_{2 l} \mathrm{Q}\left(\sqrt{a(i, M) \frac{\gamma_{g} \gamma_{i}}{\gamma_{i}+c_{2 l}^{2} \gamma_{g}}}\right)\right. \\
& \left.+(1-\alpha) \mathrm{Q}\left(\sqrt{a(i, M) \gamma_{g}}\right)\right\} .
\end{aligned}
$$

Figure 7 presents curves of BEP for 64-QAM under gated multilevel Gaussian impulsive noise for different values of signal-to-impulsive noise ratio $\gamma_{i}=\frac{E_{b}}{N_{i}}$. The probability distribution of the levels of amplitude of $C_{2}(t)$ is given by

$$
\left\{\begin{array}{l}
P\left\{C_{2}(t)=0\right\}=p_{20}=0.25 \\
P\left\{C_{2}(t)=1\right\}=p_{21}=0.25 \\
P\left\{C_{2}(t)=2\right\}=p_{22}=0.20 \\
P\left\{C_{2}(t)=3\right\}=p_{23}=0.15 \\
P\left\{C_{2}(t)=4\right\}=p_{24}=0.15
\end{array} .\right.
$$

For such case, the levels of amplitude 0 and 1 are more probable to occur than levels 3 and 4 , which have probability 0.15 . That behavior of $C_{2}(t)$ makes the modulating signal $C_{2}(t) \eta_{i}(t)$ (which represents the component of the impulsive noise) have variations of higher amplitudes with a smaller probability and small variations of amplitudes with a higher

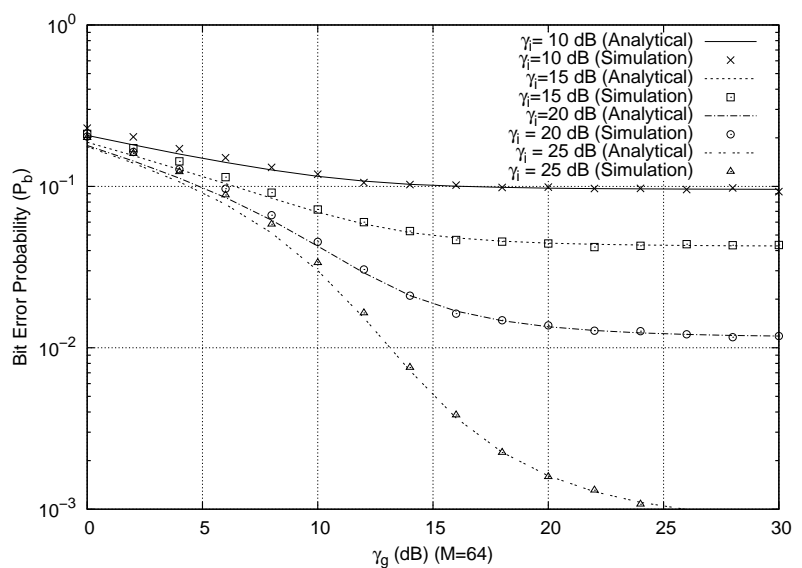

Fig. 7. BEP of 64-QAM under gated multilevel Gaussian impulsive noise.

probability. It is also observed that for $\gamma_{g}=25 \mathrm{~dB}$ the BEP obtained is $10^{-3}$ with $\gamma_{i}$ equals $25 \mathrm{~dB}$. In such case, for $\gamma_{g}=\gamma_{i}, N_{i}=N_{0}$ and since $\eta_{i}(t)$ affects the transmitted signal less frequently, it follows that the BEP can attain smaller values.

\section{BEP of the Double Gated Multilevel Gaussian Impulsive Noise}

For the double gated multilevel Gaussian impulsive noise, presented in Section VI, the probability $P_{b}(k)$ can be written as

$$
\begin{aligned}
& P_{b}(k)=\frac{2}{\sqrt{M}} \sum_{i=0}^{\left(1-2^{-k}\right) \sqrt{M}-1} w(i, k, M) \times \\
& \quad\left\{\alpha \beta \sum_{m=0}^{m_{1}-1} \sum_{n=0}^{m_{2}-1} p_{1 m} p_{2 n} \mathrm{Q}\left(\sqrt{a(i, M) \frac{\gamma_{g} \gamma_{i}}{\gamma_{i}+c_{1 m}^{2} c_{2 n}^{2} \gamma_{g}}}\right)\right. \\
& \left.\quad+(1-\alpha \beta) \mathrm{Q}\left(\sqrt{a(i, M) \gamma_{g}}\right)\right\} .
\end{aligned}
$$

In Figure 8, the BEP curves are presented for the case in which the probability of $C_{1}(t)$ assume null value is higher than the one of assuming unitary value. This is the case for which the modulated impulsive noise, with null amplitude, is more frequent. The probability distribution function of $C_{1}(t)$ and $C_{2}(t)$ for this case is

$$
\begin{gathered}
\left\{\begin{array}{l}
P\left\{C_{1}(t)=0\right\}=p_{10}=0.7 \\
P\left\{C_{1}(t)=1\right\}=p_{11}=0.3
\end{array}\right. \\
\left\{\begin{array}{l}
P\left\{C_{2}(t)=0\right\}=p_{20}=0.4 \\
P\left\{C_{2}(t)=1\right\}=p_{21}=0.2 \\
P\left\{C_{2}(t)=2\right\}=p_{22}=0.2 \\
P\left\{C_{2}(t)=3\right\}=p_{23}=0.2
\end{array}\right.
\end{gathered}
$$

It is observed in Figure 8, with respect to the curve corresponding to $\gamma_{i}=10 \mathrm{~dB}$, the decrease of BEP for $\gamma_{g}=30$ $\mathrm{dB}$. That decrease is due to the probability distribution function of $C_{1}(t)$ and $C_{2}(t)$, or more precisely, due to the fact that the probability of $C_{1}(t)=0$ is higher than the one of $C_{1}(t)=1$. 


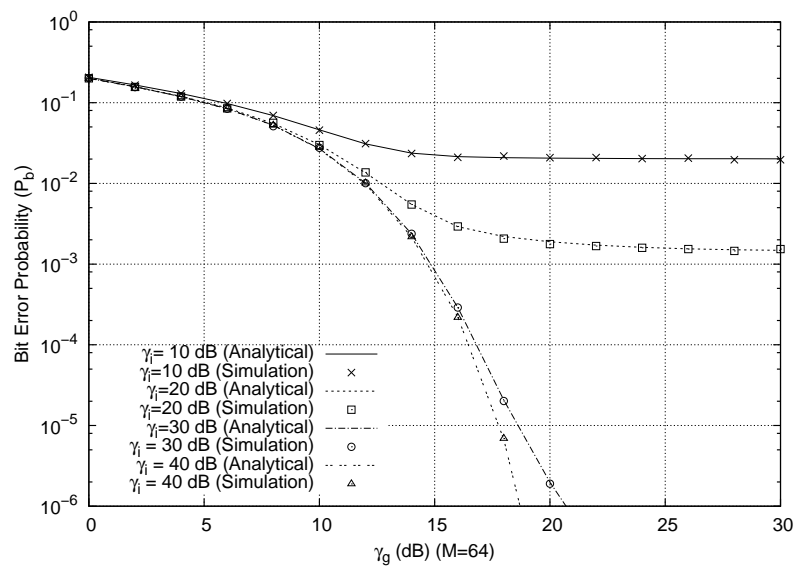

Fig. 8. BEP of 64-QAM under double gated multilevel Gaussian impulsive noise.

Additionally, in the range where $C_{1}(t)=1, C_{2}(t)$ assumes null value with probability 0.4 , which characterizes a more smooth influence of the modulated impulsive noise.

\section{CONCLUSION}

In this paper, an approach was presented for the study of gated Gaussian impulsive noise and its impact in the performance of a MAP receiver with M-QAM. In the approach, the presence or absence of the impulsive noise is characterized by an auxiliary random process which modulates the amplitude of the model AWGN $\eta_{i}(t)$. That modulation of the process $\eta_{i}(t)$ characterizes both its random variation of power and random variation of occurrence. Although the model of gated Gaussian impulsive noise already exists in the literature, its mathematical analysis by means of auxiliary processes $C_{1}(t)$ and $C_{2}(t)$ simplifies the calculus of the pdf, the autocorrelation and the power spectral density as well as the evaluation of its effects in the performance of receivers for a variety of digital modulation schemes, such as M-QAM. In this scenario, in the present paper, new exact expressions were presented for the BEP of M-QAM under impulsive noise.

As future work, one can cite the extension of the analysis of BEP presented here in order to account the fading [33] to fully characterize the effect of the gated noise in mobile communication systems.

\section{ACKNOWLEDGEMENTS}

The authors would like to express their thanks to CNPq and CAPES for the financial support of this work.

\section{REFERENCES}

[1] J. Lago-Fernández and J. Salter, "Modelling impulsive interference in DVB-T: Statistical analysis, test waveforms and receiver performance," BBC R\&D White Paper WHP, vol. 80, 2004.

[2] M. Ghosh, "Analysis of the effect of impulse noise on multicarrier and single carrier QAM systems," IEEE Transactions on Communications, vol. 44, pp. 145-147, February 1996. doi: http://dx.doi.org/10.1109/26. 486604.

[3] V. S. Vaseghi, Advanced Digital Signal Processing and Noise Reduction. John Wiley \& Sons, Second ed., 2000.

[4] W. R. Bennett, Electrical Noise. McGraw-Hill Book, 1960.
[5] A. V. Nikitin, "On the interchannel interference in digital communication systems, its impulsive nature, and its mitigation," EURASIP Journal on Advances in Signal Processing, vol. 2011, p. 137, 2011. doi: http: //dx.doi.org/10.1186/1687-6180-2011-137.

[6] A. V. Nikitin, M. Epard, J. B. Lancaster, R. L. Lutes, and E. A. Shumaker, "Impulsive interference in communication channels and its mitigation by SPART and other nonlinear filters," EURASIP Journal on Advances in Signal Processing, vol. 2012, no. 1, pp. 1-29, 2012. doi: http://dx.doi.org/10.1186/1687-6180-2012-79.

[7] M. Nassar, K. Gulati, Y. Mortazavi, and B. L. Evans, "Statistical modeling of asynchronous impulsive noise in powerline communication networks," in Proceedings of the IEEE Global Telecommunications Conference (GLOBECOM 2011), (Houston, USA), pp. 1-6, December 2011. doi: http://dx.doi.org/10.1109/GLOCOM.2011.6134477.

[8] S. Barmada, A. Musolino, M. Raugi, R. Rizzo, and M. Tucci, "A wavelet based method for the analysis of impulsive noise due to switch commutations in power line communication (PLC) systems," IEEE Transactions on Smart Grid, vol. 2, pp. 92-101, March 2011. doi: http://dx.doi.org/10.1109/TSG.2010.2099135.

[9] E. C. T. de Macêdo, D. B. Araújo, E. G. Costa, R. C. S. Freire, W. T. A. Lopes, I. S. M. Torres, J. M. R. Souza Neto, S. A. Bhatti, and I. Glover, "Wavelet transform processing applied to partial discharge evaluation," in Proceedings of the 25th International Congress on Condition Monitoring and Diagnostic Engineering (COMADEM'12), (Huddersfield, UK), pp. 1-16, June 2012. doi: http://dx.doi.org/10.1088/ 1742-6596/364/1/012054.

[10] G. Madi, F. Sacuto, B. Vrigneau, B. L. Agba, Y. Pousset, R. Vauzelle, and F. Gagnon, "Impacts of impulsive noise from partial discharges on wireless systems performance: Application to MIMO precoders," EURASIP Journal on Wireless Communications and Networking, vol. 2011, pp. 1-12, November 2011. doi: http://dx.doi.org/10.1186/ 1687-1499-2011-186.

[11] F. Yamada, F. Sukys, G. Bedicks-Junior, C. Akamine, L. T. M. Raunheitte, and C. E. Dantas, "Sistema de TV digital," Revista Mackenzie de Engenharia e Computação, vol. 5, no. 5, pp. 13-96, 2004.

[12] A. Mengi and A. J. H. Vinck, "Successive impulsive noise suppression in OFDM," in Proceedings of the 14th IEEE International Symposium on Power Line Communications and Its Applications (ISPLC 2010), (Rio de Janeiro, Brazil), pp. 33-37, March 2010. doi: http://dx.doi.org/10. 1109/ISPLC.2010.5479927.

[13] C. Akamine, F. Yamada, G. Bedicks, C. E. S. Dantas, F. Sukys, R. E. Motoyama, R. M. Maroj, G. M. Valeira, and E. L. Horta, "Field trial for Brazilian DTV using space diversity," in Proceedings of the IEEE International Symposium on Broadband Multimedia Systems and Broadcasting (BMSB 2010), (Shangai, China), pp. 1-11, 2010. doi: http://dx.doi.org/10.1109/ISBMSB.2010.5463144.

[14] M. Cotton, R. Achatz, J. Wepman, and P. Runkle, "Interference potential of ultrawideband signals part 2: Measurement of gated-noise interference to C-band satellite digital television receivers," Technical Report TR05-429, National Telecommunications and Information Administration, Boulder, Colorado, USA, 2005.

[15] W. J. L. Queiroz, W. T. A. Lopes, F. Madeiro, and M. S. Alencar, "An alternative method to compute the bit error probability of modulation schemes subject to Nakagami-m fading," EURASIP Journal on Advances in Signal Processing, vol. 2010, pp. 1-12, September 2010. doi: http://dx.doi.org/10.1155/2010/574109.

[16] N. Kim, H. Byun, Y. You, and K. Kwon, "Blind signal processing for impulsive noise channels," Journal of Communications and Networks, vol. 14, pp. 27-33, February 2012. doi: http://dx.doi.org/10.1109/JCN. 2012.6184548 .

[17] W. J. L. Queiroz, M. S. Alencar, W. T. A. Lopes, and F. Madeiro, "Error probability in multichannel reception with $M$-QAM, $M$-PAM and R-QAM schemes under generalized fading," IEICE Transactions on Communications, vol. E93-B, pp. 2677-2877, October 2010. doi: http://dx.doi.org/10.1587/transcom.E93.B.2677.

[18] W. J. L. Queiroz, F. Madeiro, W. T. A. Lopes, and M. S. Alencar, "Performance analysis of generalized QAM modulation under $\eta-\mu$ and $\kappa-\mu$ fading," EURASIP Journal on Advances in Signal Processing, vol. 2013, pp. 1-10, May 2013. doi: http://dx.doi.org/10.1186/ 1687-6180-2013-104.

[19] D. A. Guimarães and G. G. R. Gomes, "Caracterização do canal para o MI-SBTVD," Revista Telecomunicações, vol. 09, pp. 32-45, Novembro 2006.

[20] H. A. Suraweera and J. Armstrong, "Noise bucket effect for impulse noise in OFDM," Electronics Letters, vol. 40, pp. 1156-1157, September 2004. doi: http://dx.doi.org/10.1049/el:20045825. 
[21] J. L. Fernández and J.Salter, "Modelling impulsive interference in DVBT: Statistical analysis, test waveform \& receiver performance," R\&D White Paper WHP 080, BBC Reseach \& Development, London, UK, 2004.

[22] C. Akamine, Y. Iano, R. Arthur, G. Bedicks, and D. G. Caetano, "Improving DVB-T for brazilian environments," in Proceedings of the VI International Telecommunications Symposium (ITS2006), (Fortaleza, Brazil), pp. 376-380, September 2006. doi: http://dx.doi.org/10.1109/ ITS.2006.4433302.

[23] A. Hazmi, Studies in TV Digital Signal Processing: Impulsive Noise Mitigation, Repeater Loop Interference Cancellation and DVB-T Transmission in CATV Networks. PhD thesis, Tampere University of Technology, Tampere, Finland, June 2007.

[24] S. Matejka and P. Hladik, "Initial tests of DVB-T receivers on tolerance to impulsive interference," in Proceedings of the 21st International Conference Radioelektronika, (Brno, Czech Republic), pp. 1-4, April 2011. doi: http://dx.doi.org/10.1109/RADIOELEK.2011.5936471.

[25] J. Salter and J. Lago-Fernández, "DTT comparison of 64QAM(2/3) with $16 \mathrm{AM}(3 / 4)$ : Co-channel interference from PAL, echos and impulsive interference," Tech. Rep. WHP 056, BBC Reseach \& Development, London, UK, 2003.

[26] ITU, "Guidelines and techniques for the evaluation of digital terrestrial television broadcasting systems including assessment of their coverage areas," Tech. Rep. BT.2035-2, International Telecommunication Union, 2008.

[27] M. Cheffena, "Industrial wireless sensor networks: Channel modeling and performance evaluation," EURASIP Journal on Wireless Communications and Networking, vol. 2012, no. 1, pp. 1-8, 2012. doi: http://dx.doi.org/10.1186/1687-1499-2012-297.

[28] J. G. Gonzalez and G. R. Arce, "Statistically-efficient filtering in impulsive environments: Weighted myriad filters," EURASIP Journal on Applied Signal Processing, vol. 2002, no. 1, pp. 4-20, 2002. doi: http://dx.doi.org/10.1155/S1110865702000483.

[29] A. Papoulis, Probability, Random Variables, and Stochastic Processes. New York: McGraw-Hill, Third ed., 1991.

[30] B. P. Lathi, Modern Digital and Analog Communication Systems. New York, USA: Oxford University Press, Third ed., 1998

[31] J. G. Proakis, Digital Communications. New York, USA: McGraw-Hill, Second ed., 2000.

[32] K. Cho and D. Yoon, "On the general BER expression of one and twodimensional amplitude modulations," IEEE Transactions on Communications, vol. 50, pp. 1074-1080, July 2002. doi: http://dx.doi.org/10. 1109/TCOMM.2002.800818.

[33] D. I. Alves, L. Z. Costa, R. Machado, and N. R. Gomes, "Avaliação da SER do protocolo SDF para canais rádio-móveis com desvanecimento $\alpha-\mu$," Revista de Tecnologia da Informação e Codificação (RTIC), vol. 3, pp. 29-35, Dezembro 2013. doi: http://dx.doi.org/10.12721/2237-5112. v03n02a04.

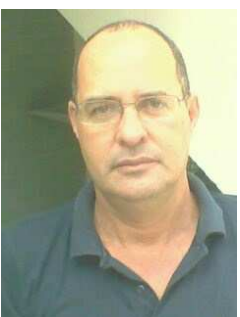

Évio da Rocha Araújo was born in Recife, PE, Brazil in 1960. He received the B.Sc. and M.Sc. degrees in Electrical Engineering from Federal University of Pernambuco, Brazil, in 1985 and 1990, respectively. He received his D.Sc. degree in Electrical Engineering from Federal University of Campina Grande, Brazil in 2013. He is with Polytechnic School of Pernambuco (POLI), University of Pernambuco (UPE) and Federal Institute of Education, Science and Technology of Pernambuco (IFPE). His research interests include wireless communication systems, communication theory, digital communications and impulsive noise.

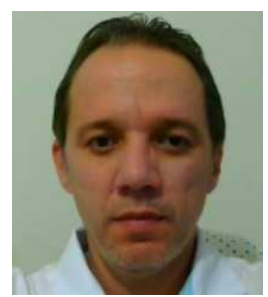

Wamberto José Lira de Queiroz was born in João Pessoa, PB, Brazil in 1973. He received the B.Sc. degree in 1997 and the M.Sc. degree in 2002 from Federal University of Paraíba and the D.Sc. degree from Federal University of Campina Grande, Campina Grande, PB, in 2004, all in Electrical Engineering. From July 2004 to January 2005 he was with University Tiradentes, Aracaju, SE. From February 2005 to January 2007, he was with University of Fortaleza, CE and from February 2007 to May 2010, he was with Federal University of Ceará. Since June 2010 he has been with the Departament of Electrical Engineering of Federal University of Campina Grande. His research interests include signal spatial processing, diversity systems, channel modelling and estimation of parameters for wireless communication. Dr. Wamberto is author of many journal and conference papers as well as a book on antenna theory and a chapter on stochastic process. He is editor of the RTIC Magazine and member of the Brazilian Telecommunications Society.

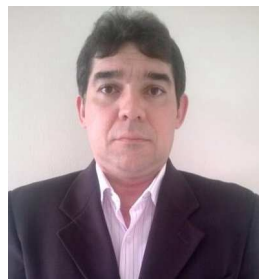

Francisco Madeiro was born in Fortaleza, Ceará, Brazil, in 1972. He received his D.Sc. degree in Electrical Engineering from Federal University of Paraíba (UFPB), in 2001. He is now with Polytechnic School of Pernambuco (POLI), University of Pernambuco (UPE), where he is Associate Professor. His main research interests include signal processing, communication systems and computational intelligence. He was recipient of the Distinguished Award in Teaching at Polytechnic School of Pernambuco, in 2008 and 2013. He was recipient of the Distinguished Award in Research at POLI, in 2013. He has been involved with $R \& D$ projects in digital transmission and image processing.

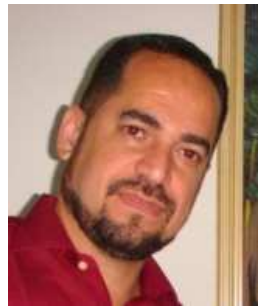

Waslon Terllizzie Araújo Lopes was born in in Petrolina, Pernambuco, Brazil in 1974. He received the B.Sc. and M.Sc. degrees in Electrical Engineering from Federal University of Paraiba, Brazil, in 1998 and 1999, respectively. He received his D.Sc. degree in Electrical Engineering from Federal University of Campina Grande, Brazil in June, 2003. Prof. Waslon Terllizzie was with AREA1 College of Science and Technology, Salvador, Brazil from August 2003 to December 2009. Until March 2015 he was with Federal University of Campina Grande and currently he is with department of Electrical Engineering of Federal University of Paraíba, João Pessoa, PB, Brazil. He is also the Executive Coordinator of the Institute for Advanced Studies in Communications (Iecom). His research interests include robust vector quantization, wireless communication systems, communication theory, and digital signal processing.

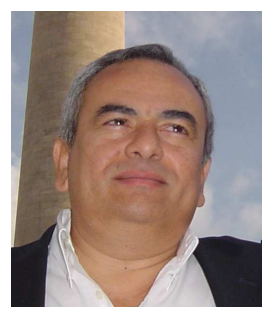

Marcelo Sampaio de Alencar was born in Serrita, Brazil in 1957. He received his Bachelor Degree in Electrical Engineering, from Universidade Federal de Pernambuco (UFPE), Brazil, 1980, his Master Degree in Electrical Engineering, from Universidade Federal da Paraiba (UFPB), Brazil, 1988 and his Ph.D. from University of Waterloo, Department of Electrical and Computer Engineering, Canada, 1994. Marcelo S. Alencar has more than 30 years of engineering experience, and 22 years as an IEEE Member, currently as Senior Member. For 18 years he worked for the Department of Electrical Engineering, Federal University of Paraiba, where he was Full Professor and supervised more than 50 graduate and several undergraduate students. Since 2003, he is Chair Professor at the Department of Electrical Engineering, Federal University of Campina Grande, Brazil. 